\title{
GENETIC RELATED CLUSTERING FOR REDUCING ENERGY CONSUMPTION IN WIRELESS SENSOR NETWORK
}

\author{
Shreya Patel $^{1}$, Jayesh Munjani ${ }^{2}$ \\ ${ }^{1}$ Student, Electronics \& Communication, CGPIT, Gujrat, India \\ ${ }^{2}$ Professor, Electronics \& Communication, CGPIT, Gujrat, India
}

\begin{abstract}
A wireless sensor network is made up of large number of mote (sensor node). Mote has limited battery power. Mote need to perform different type of task like monitor an area, sense the data, collect this data and send this data to base station. As mote (sensor node) need to do such a task it requires more battery power.so it is necessary to save energy of mote by reducing energy consumption.one can use clustering to reduce energy consumption. Clustering is grouping of mote. Energy consumption can be reduced by using intelligent hierarchical techniques for clustering. Various artificial intelligent techniques can be integrated with clustering better result can be achieved.
\end{abstract}

Key Words: Wireless sensor network, Genetic algorithm, Clustering

\section{INTRODUCTION}

Wireless sensor network is widely used in most of application like military application, home application, health application, Environment application. Mote has small power, low cost and it is multi-functional. There are different challenges like limited battery capability, transmission media, robustness, topology etc. in wireless sensor network. Battery power is necessary to keep wireless sensor network in working condition. So it is require using battery power very efficiently. For reducing battery power we can work at task level, algorithm level, protocols level. This paper concerns with hierarchical clustering strategy that produce intelligent cluster.

Cluster is simply defined as group of node. In clustering all nodes in cluster collect the data and send data to the cluster head. Each cluster has one cluster head that receives a data from its cluster member. Cluster head send this receives data to base station. Objective of clustering are set to meet the requirement for application. It is used in military application, object tracking, detection of chemical or biological agent threats, weather monitoring etc. Many objectives are load balancing, Fault tolerance, increased connectivity, reduced delay, minimal cluster count, maximum network longevity, reduce energy consumption, Scalability, localized control, reduce complexity and reduce communication overhead [1].

Clustering is depends on application, it is also affected by changes in connectivity of network; it also depends on selection of cluster head as well as distribution of cluster head. Clustering is need to be done in such way that network should work if any node die due to environment condition or due to some other reason. If any node may fail because of some reason then entire network functionality should not stop. Clustering need to be done in such way that it can maximize whole network lifetime by utilizing energy of network.
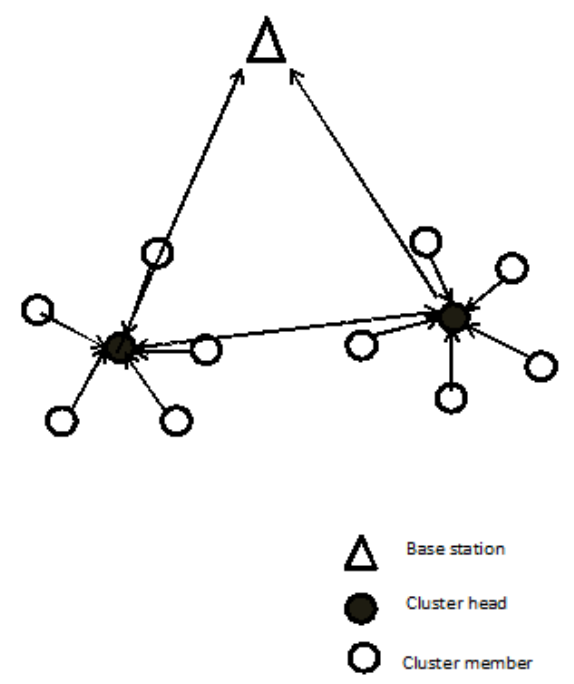

Fig -1: Basic clustering model

\section{INTRODUCTION TO GENETIC ALGORITHM}

Genetic algorithms are initiated and developed in early 1970 by John Holland. Genetic algorithms (GAs) are the main paradigm of evolutionary computing. GAs is inspired by Darwin's theory about evolution - the "survival of the fittest"[2].

The normal nodes are represented as bits of a chromosome. The cluster head and its member nodes are represented as $1 \mathrm{~s}$ and 0 s respectively [3]. A population consists of many chromosomes and the best chromosome is used to produce the next population. The fitness of the chromosome is based on the estimated energy consumption, which is determined by several fitness parameters such as distance between sensor node to sink node(Direct distance), distance between 
sensor node and cluster head(Compactness), distance between cluster head and sink node( $\mathrm{CH}$ distance), Total energy( It is sum of Energy required to send data from sensor node to cluster head, Aggregation energy and energy consume for sending a data from Cluster head to Sink node), Number of node remains in network(NT), Summation of (Remaining energy/ Initial energy).

\subsection{Operators of Genetic Algorithm}

There are three main operators of genetic algorithm. They are selection, cross over and mutation.

Selection: selection is used for creating next generation by selecting individual from old generation [2].

Crossover: According to crossover probability, it creates new generation by combining best characteristics of each parent [2].

Mutation: According to mutation probability. It exchanges a bit.

\section{CLUSTERINGUSING GENETIC ALGORITHM}

In clustering all member nodes send their data to their respective cluster head and cluster head aggregate the data and send that data to main location. in distance is reduce as all node does not send data directly to base station. As energy is directly proportional to distance, here distance is reduced so energy consumption is also reduced. Here intelligent technique is added with clustering. Genetic algorithm is added in clustering.

[1]. Initialize Population If random number is less than Optimal Election probability then assign value of chromosome as 1 otherwise 0.Optimal Election Probability of a node to become cluster headOptimal Election Probability=(Kopt /Number Of Nodes) (1)

[2]. Fitness CalculationFor this calculation, it is necessary to calculate

$>$ distance between sensor node to sink node(Direct distance)

$>$ distance between sensor node and cluster head(Compactness)

$>$ distance between cluster head and sink node $(\mathrm{CH}$ distance)

$>$ Total energy( It is sum of Energy required to send data from sensor node to cluster head, Aggregation energy and energy consume for sending a data from Cluster head to Sink node)

$>\quad$ Number of node remains in network(NT)

$>$ Summation of (Remaining energy/ Initial energy)

Fitness $=$ Direct Distance + Compactness $+\mathrm{CH}$ distance + Total Energy $+\mathrm{NT}+\operatorname{sum}($ Remaining energy/ Initial energy)

[1].Create a new population by repeating following steps until the new population is complete.

[2].Selection
[3].Tournament selection is used. In this individual which has minimum fitness is selected for next population.

[4].Crossover

[5].Two point crossover method is used. In GA optimization, crossover operation is performed with two randomly selected chromosomes decided by the crossover probability.

[6].Mutation

[7].The mutation operation involves altering the value at a randomly selected gene within the chromosome. Similarly, a mutation probability is used. Different from the crossover probability, the mutation probability is usually fairly small. In this value of zero is replaced by one.

[8].Again calculate fitness and minimum fitness value of chromosome is selected as cluster head.

[9].Go to step 2

After completing this clustering, energy of node is calculated by applying radio energy model.

\section{RESULT AND DISCUSSION}

Simulation has been done in MATLAB (2010 A) installed on Intel(R),Core (TM),i3 CPU M350@2.27 GHz having $3.00 \mathrm{~GB}$ RAM. Here for simulation $100 \times 100 \mathrm{~m}$ area is selected and 100 numbers of nodes are deployed in the network. Results have been simulated for LEACH and Genetic based clustering approach. Results also shown for the number of dead node per round, number of alive node per round, number of cluster head per round and total energy consumption per round.

Table -1: Simulation Parameter

\begin{tabular}{|c|c|}
\hline Simulation Parameter & Value \\
\hline Number of Nodes (N) & 100 \\
\hline Network size & $(50,50)$ \\
\hline Base station location & $2 \mathrm{~J}$ \\
\hline Node energy & $50 * 10^{\wedge}(-9) \mathrm{J}$ \\
\hline $\begin{array}{c}\text { Etx and Erx (Energy } \\
\text { for transmission and } \\
\text { reception) }\end{array}$ & $10^{*}\left(10^{\wedge}(-12)\right) \mathrm{J}$ \\
\hline$E_{f s}$ & $5 *\left(10^{\wedge}(-9)\right) \mathrm{J}$ \\
\hline$E_{d a}$ & $1.3 *\left(10^{\wedge}(-15)\right)$ \\
\hline$E_{a m p}$ & 5000 byte \\
\hline Data size & \\
\hline
\end{tabular}




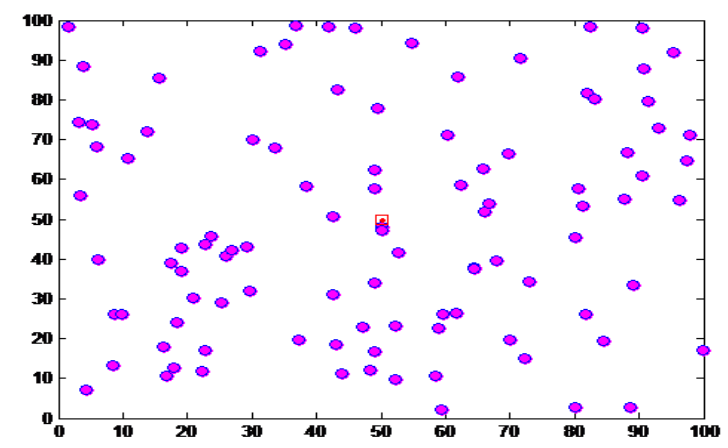

Fig -2: Location of Base station at $(50,50)$ and sensor node

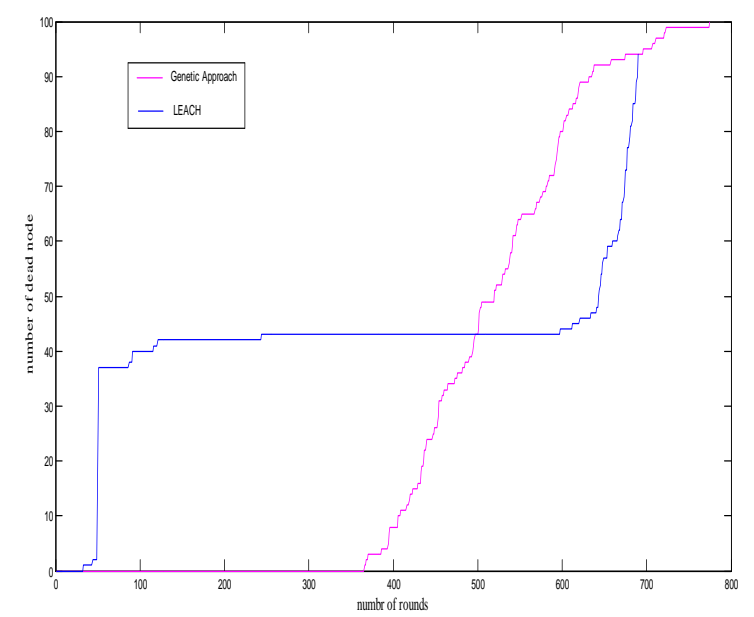

Fig -3: Number of dead node Vs Round

Figure 3 shows that first node is died after 33 round in LEACH and in Genetic based clustering first node died after 366 round. In LEACH $10 \%$ node die after 49 round and in genetic based clustering $10 \%$ node die after 408 round. Last node die after 690 rounds in LEACH and 775 rounds in Genetic based clustering.

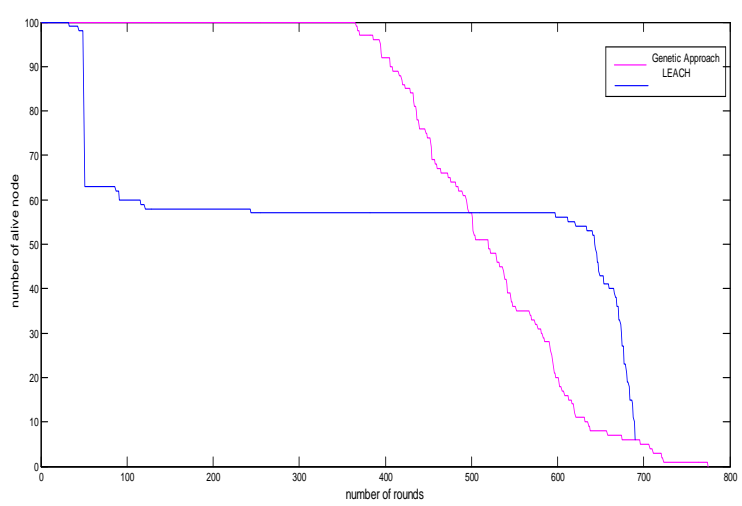

Fig -4: Number of alive node Vs Round

Figure 4 shows that 99 node is alive after 33 round in LEACH and in Genetic based clustering 99 node remain alive after 366 round.

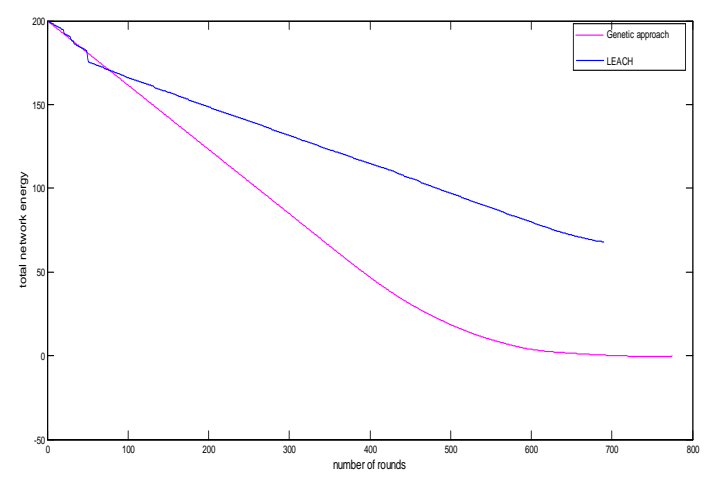

Fig -5: Total Network Energy Vs Round

Figure 5 shows that energy utilization is better in Genetic based clustering and hence network lifetime is increase using genetic approach. Genetic based Approach is better than LEACH.

Table -2: Comparison of LEACH and Genetic Approach

\begin{tabular}{|c|c|c|}
\hline Number of node die & LEACH & Genetic Approach \\
\hline First node die & 33 & 366 \\
\hline $10 \%$ node die & 49 & 408 \\
\hline $100 \%$ node die & 690 & 775 \\
\hline
\end{tabular}

\section{CONCLUSIONS}

In genetic algorithm, the nodes are represented as bits of a chromosome. The cluster head and its member nodes are represented as $1 \mathrm{~s}$ and 0 s respectively. A population having many chromosomes and the best chromosome is used to generate the next population. The fitness of the chromosome is based on the estimated energy consumption, which is determined by several fitness parameters such as distance between sensor node to sink node(Direct distance), distance between sensor node and cluster head(Compactness), distance between cluster head and sink node $(\mathrm{CH}$ distance), Total energy. Based on the fitness, the population transforms into the future generation.

In our topology Genetic based clustering is $12.31 \%$ better than LEACH. Other intelligent technique can be added in hierarchical clustering and can achieve a better result.

\section{REFERENCES}

[1]. Abbasi, A. A., \& Younis, M. (2007).“A survey on clustering algorithms for wireless sensor networks". Computer communications, 30(14), 28262841.

[2]. S. Rajasekaran, G. A. Vijayalakshmi pai "Neural networks, fuzzy logic and genetic algorithm".

[3]. Ian F. Akyildiz, Mehmet Can Vuran, "Wireless Sensor Networks", Wiley Publication,2010.

[4]. Naeimi, S., Ghafghazi, H., Chow, C. O., \& Ishii, H. (2012). "A survey on the taxonomy of cluster-based routing protocols for homogeneous wireless sensor networks". Sensors, 12(6), 7350-7409. 
[5]. Wang, J.; Zhang, Z.; Xia, F.; Yuan, W.; Lee, S. “An Energy Efficient Stable Election-Based Routing Algorithm for wirelesss sensor Networks". Sensors 2013, 13, 14301-14320.

[6]. W. R. Heinzelman, A. Chandrakasan, and H. Balakrishnan, "An Application-Specific Protocol Architecture for Wireless Microsensor Networks", IEEE Transactions on Wireless Communications, Vol. 1, No. 4,October 2002, pp. 660-670.

[7]. Sajid Hussain, Abdul W. Matin. "Hierarchical Clusterbased Routing in Wireless Sensor Networks"

[8]. Banimelhem, O.; Mowafi, M.; Taqieddin, E.; Awad, F.; Al Rawabdeh, M., "An efficient clustering approach using genetic algorithm and node mobility in wireless sensor networks," Wireless Communications Systems (ISWCS), 2014 11th International Symposium on , vol., no., pp.858,862, 26-29 Aug. 2014

[9]. Mohamed Elhoseny, Xiaohui Yuan, "Balancing Energy Consumption in Heterogeneous Wireless Sensor Networks using Genetic Algorithm" IEEE 2013.

\section{BIOGRAPHIES}

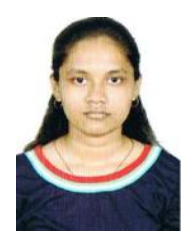

Shreya Patel, completed Master of Technology In Electronics \& Communication at Uka Tarsadia University, Bardoli, Surat 